\title{
Beta-Agonisten i.v.: Mehr Schaden als Nutzen?
}

\author{
In einer vorhergehenden Studie schien die intravenöse Infusion \\ des Beta-2-Agonisten Salbutamol für bis zu 7 Tage bei akutem \\ progressives Lungenversagen (ARDS) positive Effekte auf \\ Lungenödem und Atemdruck zu haben. Deshalb haben F. G. \\ Smith et al. diese Intervention placebokontrolliert und \\ randomisiert in einer größeren Studie untersucht.
} Lancet 2012; 379: 229-235

Zwischen Dezember 2006 und März 2010 rekrutierten die Studienautoren an 46 britischen Intensivstationen intubierte und mechanisch beatmete Patienten, die mindestens 16 Jahre alt waren. Sie erhielten randomisiert innerhalb von 72 Stunden nach Beginn der ARDS intravenös entweder $15 \mu \mathrm{g}$ Salbutamol $/ \mathrm{kg}$ standardisiertem Körpergewicht pro Stunde $(\mathrm{n}=162)$ oder Placebo ( $n=164)$ für max. 7 Tage. Die Medikation war gegenüber den Teilnehmern, dem medizinischen Fachpersonal und den Angehörigen verblindet. Als primären Endpunkt analysierten die Wissenschaftler die Mortalität innerhalb von 28 Tagen nach Randomisierung.

\section{Vorzeitiges Ende der Studie $\nabla$}

Die Rekrutierung für die Studie musste nach einer zweiten Interimsanalyse wegen Sicherheitsbedenken vorzeitig gestoppt werden. Entgegen früherer Hinweise reduzierte Salbutamol in dieser Studie nicht etwa die Mortalität, sondern erhöhte sie: 55 von 161 Patienten verstarben innerhalb von 28 Tagen in der Interventions-, 38 von 163 in der Placebogruppe(34 vs. 23\%). Dies entspricht einer relativen Risikoerhöhung um das 1,47-Fache (95\% Konfidenzintervall 1,03-2,08). Daneben reduzierten sich auch die ventilatorfreien Tage und die von Organversagen freien Tage bei Salbutamoltherapie. Die Behandlung mit dem Beta2-Agonisten wurde zudem schlecht toleriert. So traten gehäuft Tachykardie, Arrythmie und Laktatacidose auf. Auf der anderen Seite lag die Mortalität in der Placebogruppe deutlich unter dem zu Erwartenden, was die Differenz zwischen den Studienarmen erklärt. In der Literatur wird die Mortalität bei ARDS mit 40 - 60\% angegeben, hier lag die 28-Tage-Mortalität bei $23 \%$. Die Autoren vermuten, dass dies auf eine insgesamt verbesserte Behandlung bei ARDS zurückzuführen ist.

\section{Fazit}

Eine frühe intravenöse Behandlung mit Salbutamol bei ARDS wird schlecht toleriert. Nach der Interimsanalyse ist ein günstiger Effekt unwahrscheinlich, im Gegenteil: Es wurde ein negativer Effekt beobachtet. Daher kann der routinemäßige intravenöse Einsatz von Beta2-Agonisten bei ARDS nicht empfohlen werden, so die Autoren.

Friederike Klein, München
Arzneimittel

\section{Beipackzettel: Kleingedruckt, aber verständlich}

Beipackzettel von Arzneimitteln sind für Verbraucher in Deutschland besser zu verstehen als andere Gebrauchsanweisungen. Das zeigt eine aktuelle repräsentative Verständlichkeitsstudie, die das Meinungsforschungsinstitut Forsa im Auftrag der ERGO-Versicherungsgruppe AG erstellt hat. Demnach findet mehr als ein Drittel der Befragten, dass die Informationen auf dem Beipackzettel „für jeden verständlich sind“. „Das Ergebnis überrascht uns nicht“, sagt Dr. Norbert Gerbsch vom Bundesverband der Pharmazeutischen Industrie e. V. „Die Pharmaindustrie setzt sich seit Langem für die gute Verständlichkeit von Beipackzetteln ein, denn Arzneimittel sind sensible Produkte, die Risiken und Nebenwirkungen mit sich bringen können. Die von uns durchgeführten Lesbarkeitstests sind ein ganz wesentlicher Schritt, um die Verständlichkeit der Beipackzettel trotz der Komplexität der zu vermittelnden Informationen zu erhöhen. Die Texte müssen den umfangreichen gesetzlichen Anforderungen gerecht werden, v. a. aber muss der Patient sich zusätzlich zum ärztlichen Rat selbständig, ausführlich und verständlich informieren können.“

Im Rahmen der Studie hat Forsa 2600 erwachsene Bürger in Deutschland zur Verständlichkeit unterschiedlicher offizieller Schriftdokumente befragt. Dabei zeigte sich, dass insbesondere Steuer- und Versicherungsdokumente für viele schwer zu verstehen sind. Nur 5\% der Befragten sahen sich dazu als Laien in der Lage. Auch die Produktinformationen von Mobilfunkund Stromanbietern lassen zu wünschen übrig: Mobilfunkunterlagen wurden nur von $11 \%$ verstanden, Stromkundeninformationen erschlossen sich 15\% der Befragten. Das Kleingedruckte auf Lebensmittelpackungen schnitt besser ab: hier verstanden $26 \%$ der Befragten, was über die Produkte gesagt wird. Dass Beipackzettel von immerhin 32\% der Befragten als „von jedem verständlich“ eingestuft wurden, spricht für eine gute Patientenorientierung der Hersteller.

Mitteilung des Bundesverbands der Pharmazeutischen Industrie e.V., Berlin 